\title{
Transperineal Implementation of Biocompatible Balloon in Patients Treated With Radiotherapy for Prostate Carcinoma: Feasibility and Quality Assurance Study in Terms of Anatomical Stabilization Using Image Registration Techniques
}

\author{
Vassilis $\mathrm{K}^{1,2 *}$, Matsopoulos $\mathrm{G}^{2}, \mathrm{G}$ \\ $\mathrm{A}^{4}$, Kouvaris $\mathrm{J}^{4}$ and Kelekis $\mathrm{N}^{1}$ \\ ${ }^{1} 2^{\text {nd }}$ Department of Radiology, Radiotherapy Unit, ATTIKON University hospital, Xaidari, Greece \\ ${ }^{2}$ Laboratory of microwave and fiber-optics, Electrical and Computer Engineering, National Technical University of Athens, Greece \\ ${ }^{3}$ Urology Department, St. Sawwas Anticancer Hospital, Athens, Greece \\ ${ }^{4} 1^{\text {st }}$ Department of Radiology, Radiotherapy Unit, Aretaieion University, Athens, Greece
}

\begin{abstract}
The purpose of this study was to evaluate the feasibility of the transperineal implementation of biocompatible balloon (Prospace $\AA$ ) as well as the quality assurance of the procedure in terms of anatomical stabilization of the implant and reproductivity of treatment parameters during radiation therapy. Between December 2011 and February 2012, ten patients diagnosed with localized low risk prostate cancer, GS <7, PSA < 10, cT1-2, were treated with external three dimensional conformal radiation therapy (3DCRT). Before the initiation of treatment, the Prospace $®$ balloon was implanted by a minimally invasive procedure, though the perineum in the intermediate space between the rectum and the prostate. All patients underwent 3DCRT with 76-78 Gy in 38-39 daily fractions (2.0 Gy/ fraction). We evaluated the implant stabilization by examining by Computed Tomography its position right after implantation and three weeks after initiation of treatment, by using non-rigid registration technique. We also evaluated the acute toxicity according to EORTC/RTOG radiation toxicity criteria and also according to Subjective-RectoSigmoid (S-RS) scale based on the endoscopic terminology of the World Organization for Digestive Endoscopy, as well as pain related to Prospace implementation according to Visual Analogue Score (VAS). By using registration techniques, the Prospace device was found stable in sequential CTs with $x, y, z$-axis displacements up to $2.1 \mathrm{~mm}, 3 \mathrm{~mm}$ and 2.2 $\mathrm{mm}$, respectively. The mean VAS score related to Prospace was $1.4( \pm 0.5)$ and the mean score of rectal toxicity according to S-RS score was 1.9( \pm 0.6$)$. The implementation of PROSPACE is feasible. Implant's position is relative stable. The procedure is minimally invasive with no recorded side effects. The incidence of patient-reported acute Gastrointestinal $(\mathrm{Gl})$ and Genitourinary $(\mathrm{GU})$ toxicity as well as findings from flexible rectosigmoidoscopy, following high dose of 3DCRT after the prospace implantation, were low. The study is on-going and further results with analyzed data from more patients will be reported.
\end{abstract}

Keywords: Prostate cancer; Biocompatible balloon; Implementation; Radiotherapy; Radiation toxicity; Image registration techniques

Abbreviations: EBRT: External Beam Radiation Therapy; 3DCRT: 3 Dimensional Conformal Radiation Therapy; IMRT: Intensity Modulated Radiation Therapy; GI: Gastro - Intestinal; GU: Genito Urinary; VAS: Visual Analogue Score; S-RS: Subjective-RectoSigmoid; PSA: Prostate Specific Antigen; TRUS: Transrectal Ultra Sound; IC: Isocenter; CB: Centroid Balloon

\section{Introduction}

One of the standard curative treatments for patients suffering from low risk prostate carcinoma is radical External Beam Radiation Therapy (EBRT). Alone or in combination with hormonal therapy, its therapeutic results are comparable to radical prostatectomy. EBRT can be delivered with either three Dimensional Conformal Radiotherapy (3DCRT) or Intensity Modulated Radiation Therapy (IMRT) technique [1].

There is a definite relationship between dose escalation and response to radiation treatment with radiation induced morbidity to normal surrounding tissues [2-6]. IMRT improves the treatment outcome, sparing the normal surrounding tissues and reducing the acute and late radiation induced toxicity [4-6]. The total dose of radiotherapy that can be delivered through conventional conformal techniques is still limited by the tolerance of surrounding normal tissues, mainly the rectum and the bladder [7].

Levy et al. in 2009 reported on a new balloon made of a biodegradable polymer called Prospace ${ }^{\bullet}$ consisted of polylactideco-epsilon-caprolactone [8]. Balloon's mechanical and chemical properties were documented both in vitro and in vivo. Prospace was found safe and effective for its intended use of separating prostate from the rectum for a desired duration in experimental models [9].

\section{Materials and Methods}

\section{Patient characteristics}

Ten selected patients were included in this study. All patients

*Corresponding author: Vassilis K, Medical School, National University of Athens, Greece, Fax: 00302105326418; E-mail:vkouloul@ece.ntua.gr

Received March 29, 2013; Accepted April 18, 2013; Published April 25, 2013

Citation: Vassilis K, Matsopoulos G, Georgakopoulos J, Kalogeropoulos T, Platoni K, et al. (2013) Transperineal Implementation of Biocompatible Balloon in Patients Treated With Radiotherapy for Prostate Carcinoma: Feasibility and Quality Assurance Study in Terms of Anatomical Stabilization Using Image Registration Techniques. J Bioequiv Availab 5: 142-148. doi:10.4172/jbb.1000149

Copyright: ( 2013 Vassilis K, et al. This is an open-access article distributed under the terms of the Creative Commons Attribution License, which permits unrestricted use, distribution, and reproduction in any medium, provided the original author and source are credited. 
Citation: Vassilis K, Matsopoulos G, Georgakopoulos J, Kalogeropoulos T, Platoni K, et al. (2013) Transperineal Implementation of Biocompatible Balloon in Patients Treated With Radiotherapy for Prostate Carcinoma: Feasibility and Quality Assurance Study in Terms of Anatomical Stabilization Using Image Registration Techniques. J Bioequiv Availab 5: 142-148. doi:10.4172/jbb.1000149

were suffering from low or intermediate risk prostate carcinoma for which they underwent 3DCRT at Attikon University Hospital of Athens, between December 2011 and February 2012. The pretreatment evaluation included medical history, physical examination, blood tests (including Complete Blood Count, Prostate Specific Antigen level PSA, liver and renal function tests) and imaging exams with Computed Tomography (CT) and/or Magnetic Resonance (MRI) of the pelvis. The patient characteristics are summarized in table 1.

\section{Inclusion-exclusion criteria}

Eligible patients had histologically confirmed clinical Prostate Cancer Stage T1-2 (according to American Joint Committee on Cancer staging manual, 7th edition, 2010), Gleason Score $<7$ and PSA levels less than $10 \mathrm{ng} / \mathrm{mL}$. All patients had good performance status according to Eastern Cooperative Oncology Group Performance score of 0-1.

Patients were ineligible if they had undergone previous pelvic RT, previous history of any rectal disease or surgery to pelvis, neoadjuvant androgen ablative therapy, lymph node metastatic involvement, distant metastases or had a hip prosthesis. Patients with cardiovascular or coagulation problems were also excluded from the study.

\section{Balloon implementation}

In all patients Prospace ${ }^{\bullet}$ balloon was implanted though the perineum [9]. The Prospace ${ }^{\oplus}$ Balloon implantation was performed in an outpatient basis under local anesthesia. Prior to the implantation each patient was examined with blood tests and urine culture to ensure that he has no urine infection. Patients were also checked for coagulation disorders in order to stop any anticoagulant therapy 5 days prior to the implantation date. Prophylactic antibiotic therapy per-os was administered the same day of the procedure preferably such as a fluoroquinolone, together with a rectum enema.

The Prospace Balloon system packaging consisting of a biodegradable inflatable balloon mounted on a deployer and a balloon delivery echogenic kit consisting of a needle, a dilator and an introducer sheath. The balloon material was a co-polymer of poly lactide acid and epsilon caprolactone which was designed to degrade in situ after 3-6 months from placement. A syringe was also required to perform skin and fascia anesthesia and to inflate the balloon with warm saline.

Placement of the balloon was performed under local anesthesia and continuous Trans Rectal Ultra Sound (TRUS) guidance. The patient was placed in the lithotomy position and the perineum was scrubbed in a standard manner, while a urethral catheter was inserted and kept in place during the whole procedure. A biplane TRUS probe was inserted in patient's rectum and kept in place throughout the whole procedure. Local anesthesia of the perineum was performed by an anesthetic solution injected in the midline approximately $1,5 \mathrm{~cm}$ above the anal verge. After local anesthesia of the perineum skin and underlying fascia has been achieved, the needle of the delivery kit was inserted in the same location of the local anesthesia and was advanced until the

\begin{tabular}{|c|c|}
\hline Median age (range) & $71(65-77)$ \\
\hline Mean initial PSA (range) & $9.1 \pm 0.8 \mathrm{ng} / \mathrm{ml}(7.2-9.8)$ \\
\hline T1 & $3 / 10$ \\
\hline T2 & $7 / 10$ \\
\hline
\end{tabular}

Table 1: Patient characteristics. prostate apex under continuous TRUS guidance. From this point, we continue to advance the needle while making hydro dissection with the anesthetic solution, until the needle reaches the prostate base. The hydro dissection with the anesthetic solution produces local anesthesia but also facilitates the separation of the space between the rectum and the prostate and will enable smoother entry and advancement of the introducer sheath later in the procedure. When the needle reaches the correct position, verified by the TRUS, we made a perineal skin and fascia incision, $0.5 \mathrm{~cm}$ high and $1 \mathrm{~cm}$ deep around the needle access point. The incision was done to allow room for the dilator and the introducer sheath to be inserted freely into the perineum. Next, while holding the needle in place we advanced the dilator, coupled with the introducer sheath, over the needle towards the prostate base until the designated mark on the needle appears. At this point the dilator tip was aligned with the needle tip and while holding firmly the dilator and introducer sheath in place, we remove the needle. Then, while holding the dilator in place, we continue to advance the sheath over the dilator until the sheath reaches the prostate base as viewed by the TRUS. After verifying the correct position of the sheath in the midline by TRUS, we holded firmly in place the introducer sheath and removed the dilator leaving the introducer sheath in place. Now the introducer sheath acted as a working channel through which the balloon would be introduced. To introduce the balloon, we holded the introducer sheath firmly and we insured that the balloon deployer centering strip was facing upwards while advancing the balloon through the introducer sheath until the mark on the deployer reaches the introducer's sheath proximal end. Then to withdraw the introducer sheath we holded the balloon deployer firmly and we pulled the introducer sheath all the way back. At this point the balloon was fully exposed in situ and ready for deployment. Once again we verified by TRUS that the balloon was correctly positioned in the midline. We slowly started to fill the balloon with warm saline under continuous TRUS visualization, in order to ensure that during the inflation of the balloon the rectal wall remained at least $3 \mathrm{~mm}$ thick (Figure 1). The volume of the saline inflated into the balloon, was unique for each balloon, and marked on the package of the balloon, but usually was between $14 \mathrm{cc}$ to $17 \mathrm{cc}$ of normal saline. Once the balloon was fully inflated with the designated amount of saline, we detached and sealed the balloon in place by firmly retracting the inflation syringe from the deployer. Now the balloon was firmly positioned in place between rectum and prostate and we could

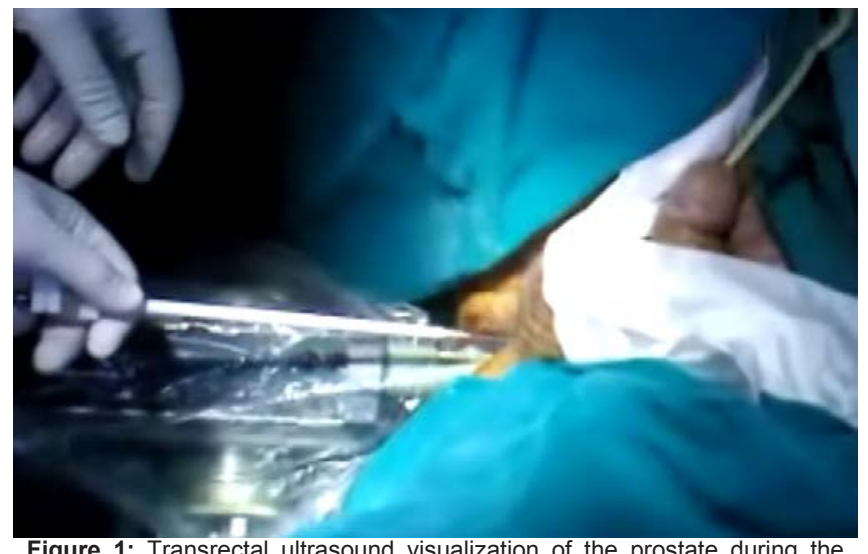

Figure 1: Transrectal ultrasound visualization of the prostate during the balloon implantation procedure and transperineal placement of the introducer sheath under ultrasound guidance. 
Citation: Vassilis K, Matsopoulos G, Georgakopoulos J, Kalogeropoulos T, Platoni K, et al. (2013) Transperineal Implementation of Biocompatible Balloon in Patients Treated With Radiotherapy for Prostate Carcinoma: Feasibility and Quality Assurance Study in Terms of Anatomical Stabilization Using Image Registration Techniques. J Bioequiv Availab 5: 142-148. doi:10.4172/jbb.1000149

remove safely the deployer and introducer sheath from the patient. We performed a final check of the balloon position by the TRUS as well as by palpation of the rectum to ensure not only that the balloon was in the correct place but also to ensure the integrity of the rectal mucosa. Finally we sutured the perineum incision, if required, and we removed the urethral catheter. The patient was dismissed from the hospital the same day, as soon as he urinates, and was given oral antibiotics for 3-5 days. The clinical use of Prospace was recently approved by the Central Board of Health of the Greek Ministry of Health. Beyond this, all patients signed informed consent about the use of Prospace.

The evaluation of pain or discomfort related to the Prospace implementation was done with the Visual Analogue Score (VAS).

\section{Radiotherapy}

Implant's position was tested right after the procedure and two or three weeks after initiation of 3DCRT by a virtual CT-simulation of the pelvis with a $0.3-\mathrm{cm}$ spacing between slices, in supine position, using "knee sponge" to consistently align thighs. Patients were instructed to have a full bladder and empty rectum (following a dietary suggestion) during simulation and the whole course of treatment thereafter. The exact position of the balloon was tested during the first CT. After acquisition of the second CT scan, possible movement of the balloon according to $\mathrm{x}, \mathrm{y}, \mathrm{z}$ axis was evaluated and measured. For the registration we used as stable the referential skin markers and the isocenter point. The balloon surface was outlined in CT and its anatomical position was monitored assessing any geographical displacement. The non-rigid registration in sequential CT scans of the pelvis revealed the stability of the Prospace devise, showing displacements only at $3 \mathrm{~mm}$ maximum.

All contouring of target volumes and normal structures (organs at risk-OARs) were performed in the Prosoma System. The following structures were delineated: balloon, clinical target volume (CTV), planning target volume (PTV) according to the International Commission on Radiation Units and Measurements (ICRU) criteria [10-12].

The CTV was the prostatic gland; the PTV was obtained by expanding CTV with a margin of $1 \mathrm{~cm}$ in each direction, and of $0.7 \mathrm{~cm}$ posteriorly. Rectum was manually contoured from the distal ischiatic branch to the sigmoid flexure. In addition bladder wall and femoral heads were contoured. The CTV, PTV and OARs were outlined on all CT slices [13]. None of our patients received pelvic node or seminal vesicles irradiation. The prescription dose of 76-78 Gy was defined for the $95 \%$ isodoses of the PTV. Beams were conformally shaped around the PTV and partial wedging or dynamic Multi Leaf Collimator (MLC) was employed to improve dose homogeneity. To evaluate the dose constraints for normal tissues we used the NCCN 2010 guidelines (www. nccn.org), the Radiation Oncology Group (RTOG) GU consensus as reported by Lawton et al. and the QUANTEC report [14,15].

The PTV was treated, using a four field technique with $15 \mathrm{MV}$ $\mathrm{X}$-ray energy beams. Weighted beams and wedges were used as necessary. The fields were placed isocentrically. Dose calculation was performed and normalized to isocenter. The total prescribed dose was 76-78 Gy, delivered in 38-39 daily fractions (Monday to Friday) to the whole prostate, given in 2 Gy fractions. Dose calculations were performed using the treatment planning system Eclipse (Release 6.5,
Varian Associates, Palo Alto, CA), to deliver the prescribed dose to the ICRU reference point with a minimum dose of $95 \%$ and a maximum dose of $107 \%$ to the PTV.

\section{Displacement measurements with registration techniques}

Two CT scans were performed: right after the implementation (treatment planning CT) and 3 weeks after the initialization of radiation treatment (verification $\mathrm{CT}$ ). After the acquisition of the two CT scans, an image registration method was employed for the spatial alignment of the $\mathrm{CT}$ volumes. The $\mathrm{CT}$ scan used for treatment planning was defined as the reference image, whereas the verification CT scan was the image to be registered. The two CT scans were aligned using an automatic point-based registration procedure. The registration procedure could be divided into 3 steps:

- Automatic extraction of landmark points in the planning CT scan.

- Correspondence establishment between homologous points of the two CT scans using an automatic iterative algorithm.

- Registration of the CT scans using the detected point correspondences.

Prior to the detailed description of the registration procedure, some notations should be introduced. Let $I_{R}$ and $I_{F}$ be the reference CT of the radiotherapy planning and the verification CT, respectively, $\mu_{A}(I)$ is the restriction of image I in region $A \subset \mathfrak{R}$ and $T(A) \subset \mathfrak{R}^{3}$ denotes a local image transformation $\mathrm{T}$ acting only on the region $\mathrm{A}$ of image $\mathrm{I}$.

The extraction of landmark points was performed automatically in each CT slice of the reference image $I_{R}$ of the radiotherapy planning CT scan using the method proposed by Rohr [16]. Points of high intensity variations were identified from the local maxima of the quantity $R$, calculated at each pixel $(x, y)$ of the $s$-slice $I_{s}$ of the reference image as follows:

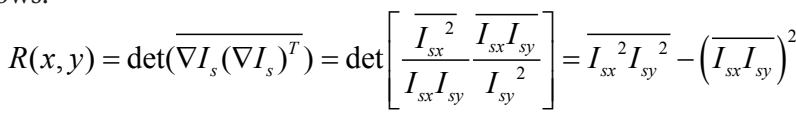

Where, the overline means average over a local neighbourhood,

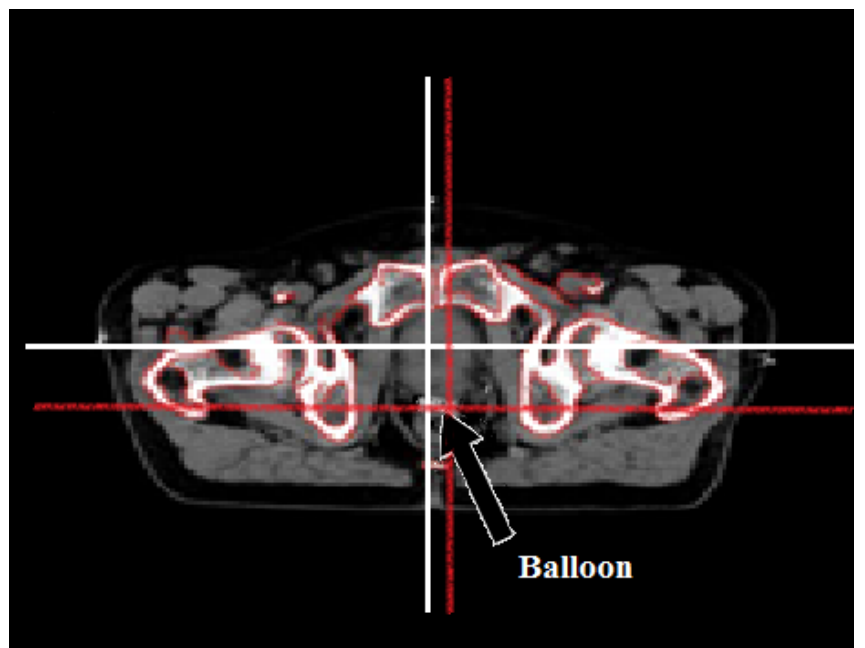

Figure 2: Abdominal $\mathrm{CT}$ plane for treatment planning purposes. The displacement between the IC (while lines) and CB (red lines) in sequential CTs was assessed after image registration. The Prospace is pointed by the white arrow. 
Citation: Vassilis K, Matsopoulos G, Georgakopoulos J, Kalogeropoulos T, Platoni K, et al. (2013) Transperineal Implementation of Biocompatible Balloon in Patients Treated With Radiotherapy for Prostate Carcinoma: Feasibility and Quality Assurance Study in Terms of Anatomical Stabilization Using Image Registration Techniques. J Bioequiv Availab 5: 142-148. doi:10.4172/jbb.1000149

the subscripts denote partial derivatives of the slice $I_{s}$ with respect to the spatial coordinates $x, y, s=1,2, \ldots, M$ denotes each slice of the reference CT $I_{R}$ and $M$ is the total number of slices. Since bony structures undergo less deformation than other anatomical features, e.g. soft tissues or organ edges, landmarks located on bones can be more accurately detected in consecutive CT scans. An appropriate threshold, defined after experimentation, was applied to the Hounsfield units of the image at the extracted landmark points, so that only landmark points located on bones were selected. In figure 2, a typical slice of abdominal CT scan is shown along with the landmark points extracted delineating the red lines around the bony structures.

For the establishment of point correspondences, an automatic iterative point correspondence algorithm was applied to the two CT volumes. The algorithm was an extension to $3 \mathrm{D}$ of the procedure proposed by Markaki et al. for detecting point correspondences in 2D medical images [17]. In particular, local rigid transformations $\mathrm{Ti}(\mathrm{Ai})$ were defined, acting only on image blocks $A i$ centered at the extracted landmark points $P i, \quad i=1,2, \ldots, N$, of the reference CT scan $I_{R}$. During the iterative procedure, candidate solutions were tested for the parameters of each local transformation $T_{i}$, which mapped the region $A i$ of the reference CT scan $I_{R}$ to corresponding regions $T i(A i)$ of the floating (verification) CT scan $I_{F}$. The candidate solutions for the transformation parameters were generated by a random number generator following a "simulated annealing" optimization scheme [18]. Thus, an optimization of the similarity between the corresponding image regions $A i$ and $T i(A i)$ in the reference and the verification CT scan was attempted iteratively. The similarity between the restrictions of the two CT volumes $I_{R}$ and $I_{F}$ to the corresponding regions $A$ and $T(A)$ was quantified by the correlation coefficient $C C$ as follows:

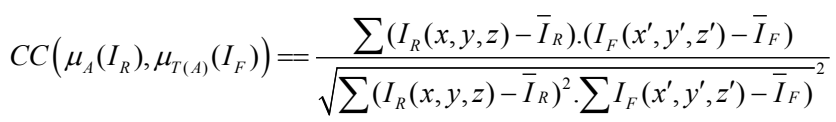

Where, $\left(x^{\prime}, y^{\prime}, z^{\prime}\right) \in T(A)$ are the transformed spatial coordinates of $(x, y, z) \in A$ and the overline means average over the specific image region $A$ or $T(A)$.

The type of transformation used to describe the spatial mapping between the CT scans was a non-rigid transformation, based on the thin plate splines (TPS) model [19]. The transformation parameters were determined by the corresponding point sets, detected during Step 2 of the procedure. The whole number of corresponding points was used for the determination of the TPS transformation parameters. In figure 2, there are two reference points derived from the intersection of the horizontal and vertical axis: the isocenter one coming from the white axis (IC) and the centroid of the balloon coming from the red axis (CB). The delineation of the balloon in all CT-slices was made manually. The edges of the reference CT slice are superimposed on the aligned CT slice of the verification scan, so that the registration results can be visually assessed. After registration, the differences between IC and $\mathrm{CB}$ at the two time points of CTs (balloon implementation and during radiotherapy) were assessed.

\section{Results}

The implementation of Prospace was feasible. Neither any serious morbidity for the patient nor any difficulty for the urologist who implemented the device was noted. The mean VAS score related to Prospace implementation was minimal at the level of $1.4(\mathrm{SD} \pm 0.5)$.

\begin{tabular}{|c|c|c|c|}
\hline Patient & $\mathbf{X}$ & $\mathbf{Y}$ & $\mathbf{Z}$ \\
\hline 1 & 2 & 2 & 3 \\
\hline 2 & 2 & 3 & 3 \\
\hline 3 & 3 & 3 & 2 \\
\hline 4 & 3 & 4 & 3 \\
\hline 5 & 2 & 2 & 2 \\
\hline 6 & 1 & 4 & 2 \\
\hline 7 & 2 & 2 & 1 \\
\hline 8 & 3 & 3 & 2 \\
\hline 9 & 2 & 5 & 2 \\
\hline 10 & $2.1 \pm 0.7$ & $3.0 \pm 1.1$ & $2.2 \pm 0.6$ \\
\hline Mean value ( \pm SD) & 1 & 2 & \\
\hline
\end{tabular}

Table 2: $\mathrm{CB}$ displacements with reference to IC concerning sequential abdominal CTs.

\begin{tabular}{|c|c|c|c|}
\hline & Grade 0 & Grade I & Grade II \\
\hline \multirow[t]{2}{*}{$\begin{array}{l}\text { EORTC-RTOG } \\
\text { scale for lower } \\
\text { Gastro-intestinal }\end{array}$} & None & $\begin{array}{l}\text { Increased frequency } \\
\text { or change in quality } \\
\text { of bowel habits not } \\
\text { requiring medication/ } \\
\text { rectal discomfort not } \\
\text { requiring analgesics }\end{array}$ & $\begin{array}{l}\text { Diarrhea requiring } \\
\text { parasympatholytic drugs/ } \\
\text { mucous discharge not } \\
\text { necessitating sanitary } \\
\text { pads/ rectal or abdominal } \\
\text { pain requiring analgesics }\end{array}$ \\
\hline & $9 / 10$ & $1 / 10$ & - \\
\hline \multicolumn{4}{|l|}{$\begin{array}{l}\text { EORTC-RTOG } \\
\text { scale for } \\
\text { Genitourinary }\end{array}$} \\
\hline \multirow[t]{2}{*}{ Nocturia } & None & 2-3 times & 4-6 times \\
\hline & $9 / 10$ & $1 / 10$ & - \\
\hline \multirow[t]{2}{*}{ Frequency } & None & Once/2 hour & Once/1 hour \\
\hline & $8 / 10$ & $2 / 10$ & - \\
\hline \multirow[t]{2}{*}{ Hematuria } & None & Microscopic & $\begin{array}{l}\text { Intermittent } \\
\text { moderate) }\end{array}$ \\
\hline & $10 / 10$ & - & - \\
\hline \multirow[t]{2}{*}{ Dysouria } & None & Slight & Moderate \\
\hline & $8 / 10$ & $1 / 10$ & $1 / 10$ \\
\hline
\end{tabular}

Table 3: EORTC/RTOG GI and GU acute radiation induced toxicity.

Some of the patients complained only for a mild discomfort for 24 hours after the implementation. The displacements of the $\mathrm{CB}$ by reference with the IC point are shown in table 2.

The median follow-up was 6 months. Treatment compliance was excellent. According to EORTC/RTOG scale, as shown in table 3, the acute toxicities were as follows: grade I GI in one patient; for GU, one patient with grade I of nocturia, two patients with grade I of frequency, one patient with grade I and one patient with grade II of dysouria. The mean score of rectal toxicity according to S-RS score was 1.9 (SD \pm 0.6 ); only grade I toxicity was noted. The results in details concerning the S-RS score are shown in table 4 . The scores of VAS, S-RS and PSA (before and post RT) are shown in table 5.

Acute toxicity was minimal after patients finished RT; at 3 months of follow up, no patient had any GI toxicity score, while two patients remained with grade I GU toxicity (dysouria). The decrease of mean score of EORTC/RTOG acute toxicity at three months compared to the score noted during irradiation was significant $(\mathrm{P}<0.01$, Wilcoxon test). 
Citation: Vassilis K, Matsopoulos G, Georgakopoulos J, Kalogeropoulos T, Platoni K, et al. (2013) Transperineal Implementation of Biocompatible Balloon in Patients Treated With Radiotherapy for Prostate Carcinoma: Feasibility and Quality Assurance Study in Terms of Anatomical Stabilization Using Image Registration Techniques. J Bioequiv Availab 5: 142-148. doi:10.4172/jbb.1000149

\begin{tabular}{|c|c|}
\hline & Grade 1 \\
\hline Subjective & \\
\hline Tenesmus & Occasional urgency \\
\hline Mucosal loss & Occasional \\
\hline & $2 / 10$ \\
\hline Sphincter control & Occasional \\
\hline & - \\
\hline Stool frequency & $2-4$ per day \\
\hline & $5 / 10$ \\
\hline Pain & Occasional \& minimal \\
\hline & $7 / 10$ \\
\hline Objective & Occult \\
\hline Bleeding & - \\
\hline & Localized-spotted congested mucosa \\
\hline Mucosa-surface & $2 / 10$ \\
\hline & Superficial $\leq 1 \mathrm{~cm}^{2}$ \\
\hline Ulceration & $2 / 10$ \\
\hline & \\
\hline
\end{tabular}

Table 4: Rectal toxicity according to S-RS grading scale at the completion of RT. Only Grade I acute toxicity was noted.

In terms of SRS score, two patients remained with only rectosigmoid findings of grade I toxicity (localized-spotted congested mucosa), showing also a significant reduction compared to the score noted at the completion or RT $(\mathrm{P}<0.01$, Wilcoxon test $)$.

Treatment was administered only in cases of GU toxicity nonsteroid anti-inflammatory for dysuria, urgency, frequency, nocturia.

\section{Discussion}

According to the literature, there is a significant correlation of dose escalation and response to treatment for prostate carcinoma $[2,3,5,7]$. Nevertheless higher doses of radiation to prostate gland result to higher doses to anterior rectal wall and the probability of severe late GI toxicity (GR3 or 4) increases. High dose of irradiation and associated higher doses to rectum have evoked the need for improved treatment planning and minimized radiation dose or protection of the rectum during prostate radiation therapy [6,7]. Radiotherapy side effects in general include rectal irritation and bleeding, erectile dysfunction and urinary frequency. Since irradiation isodose distribution includes a part of the rectum, displacing irradiated prostate away from anal and rectum, would reduce damage and therefore side effects.

It is also well known from relative literature that normal organ motion results in inter- and intra- fraction movement of prostate and seminal vesicles. One of the main contributor factors for this motion is differences in rectal volume and rectal diameter, which can lead to displacements of prostate in the range of $0.5 \mathrm{~cm}$ in cranial - caudal directions and to $1 \mathrm{~cm}$ or even $1.5 \mathrm{~cm}$ in lateral directions [20-22]. Researchers from many different institutions have tried to face these challenges: to minimize the rectal exposure to dose escalated radiation therapy and minimize also the normal movement of the prostate for reproducing prostate position, with the use of rectal balloons.

D'Amico et al. reported on the efficacy of a special designed inflatable intrarectal balloon for reducing prostate mobility, in ten patients that underwent prostate brachytherapy. They reported that the device led to minimum organ displacement while it was well tolerated by all patients [23]. Wachter et al. also tested intrarectal inflated balloon in ten patients underwent radiotherapy for prostate carcinoma. They found that the use of the balloon resulted in significant reduced displacements of the prostate and better treatment parameters in all patients [24]. Several other studies have evaluated the efficacy of rectal balloons in terms of improved radiation treatment parameters [25,26]. Tolerance of rectal balloon was also tested in a study from Ronson et al. In a large series of 3561 patients, $97.6 \%$ of patients tolerated the device well throughout radiation treatment [27].

Trying not only to minimize prostate movement during treatment but also to reduce rectal exposure to high radiation dose, many investigators tested in vivo or in vitro the use of intraperineum implanted substances or devices like balloons in order to increase the distance between anterior rectal wall and prostate gland.

Prada et al. reported in 2007 on 27 patients in whom Hyalouronic Acid (HA) was injected in perirectal fat to increase the recto-prostatic distance before radiotherapy (external beam radiotherapy and

\begin{tabular}{|c|c|c|c|c|c|}
\hline No & VAS score (Prospace) & $\begin{array}{c}\text { S-RS score } \\
\text { (end of RT) }\end{array}$ & S-RS score (3months post RT) & Baseline PSA & Post RT PSA \\
\hline 1 & 2 & 2 & 0 & 8.7 & 0.3 \\
\hline 2 & 1 & 2 & 0 & 9.8 & 0.2 \\
\hline 3 & 2 & 2 & 0 & 9.1 & 0.2 \\
\hline 4 & 1 & 3 & 1 & 9.7 & 0.1 \\
\hline 5 & 2 & 1 & 0 & 9.5 & 0.01 \\
\hline 6 & 1 & 2 & 0 & 9.6 & 0.1 \\
\hline 7 & 1 & 2 & 0 & 7.2 & 0.1 \\
\hline 8 & 2 & 2 & 0 & 9.4 & 0.1 \\
\hline 9 & 1 & 2 & 1 & 8.7 & 0.0 \\
\hline 10 & 1 & 1 & 0 & 8.9 & 0.1 \\
\hline
\end{tabular}

Table 5: VAS score related to Balloon implementation, S-RS score with subjective and objective findings (rectosigmoidoscopy) at the completion of RT and 3 months thereafter, and PSA values before and after RT. 
Citation: Vassilis K, Matsopoulos G, Georgakopoulos J, Kalogeropoulos T, Platoni K, et al. (2013) Transperineal Implementation of Biocompatible Balloon in Patients Treated With Radiotherapy for Prostate Carcinoma: Feasibility and Quality Assurance Study in Terms of Anatomical Stabilization Using Image Registration Techniques. J Bioequiv Availab 5: 142-148. doi:10.4172/jbb.1000149

brachytherapy). They concluded that injecting HA is a safe and well tolerated procedure, achieving significant decreased rectal dose during radiotherapy [28]. In another recent study from the same institution, HA was again tested in 69 consecutive patients that underwent brachytherapy for prostate carcinoma. The investigators again reported excellent results with minimal rectal toxicity, based and on endoscoping findings [29]. They also reported that the implanted or injected substance was pretty stable for many months, usually as tested by consecutive exams.

As far as transperineally implanted balloons like in our series, Levy et al. reported on the feasibility of Prospace as an implantable, biodegradable, inflatable, preshaped triangular balloon of commercially used poly (L-lactide-co-epsilon-caprolactone) co-polymer material which can provide separation between prostate and rectum [8]. Biocompatibility and degradability of the Prospace in conjunction with local irradiation were evaluated in several in vivo studies [9]. The device was found to be biocompatible in subcutaneously implanted experimental animals: up to 42 days in rabbits, up to 12 months in a transperineally implanted dog, and up to 6 months in 8 transperineally implanted balloons in pigs. According to this study, since the balloon has been inflated, it remained sufficiently stable for several months and subsequently the tissues remained separated. In all experimental animals, histopathology has shown no systemic or local toxicity. After three months post radiotherapy evaluation in pigs that received $15 \mathrm{~Gy}$ (3 fractions once per week) the investigators documented the stability of the balloon position without any local or systemic side effects. They also reported that the balloon's preparation ensures no bonding across anatomical interfaces by means of mechanical stability of implantation.

Our results are in accordance to the findings from the above mentioned studies. The implant was found stable. The image registration techniques showed a minimal displacement up to $3 \mathrm{~mm}$ in sequential CTs.

As far as VAS score, none of our patients experienced symtoms of pain affecting their performance status. In conclusion, the Prospace implementation is feasible and the radiation induced toxicity especially for the rectum is minimal, equivalent to IMRT techniques. More patients are needed for the confirmation of the results of the present study.

\section{Acknowledgements}

All authors wish to tank Professor Uzunoglou for the use of image registration techniques for the quality assurance of the balloon implementation.

\section{References}

1. Hummel S, Simpson EL, Hemingway P, Stevenson MD, Rees A (2010) Intensity-modulated radiotherapy for the treatment of prostate cancer: a systematic review and economic evaluation. Health Technol Assess 14: 1-108, iii-iv.

2. Zelefsky MJ, Fuks Z, Happersett L, Lee HJ, Ling CC, et al. (2000) Clinical experience with intensity modulated radiation therapy (IMRT) in prostate cancer. Radiother Oncol 55: 241-249.

3. Zelefsky MJ, Fuks Z, Hunt M, Lee HJ, Lombardi D, et al. (2001) High dose radiation delivered by intensity modulated conformal radiotherapy improves the outcome of localized prostate cancer. J Urol 166: 876-881.

4. Alicikus ZA, Yamada Y, Zhang Z, Pei X, Hunt M, et al. (2011) Ten-year outcomes of high-dose, intensity-modulated radiotherapy for localized prostate cancer. Cancer 117: 1429-1437.

5. Pollack A, Hanlon A, Horwitz EM, Feigenberg S, Uzzo RG, et al. (2003)
Radiation therapy dose escalation for prostate cancer: a rationale for IMRT World J Urol 21: 200-208.

6. Zelefsky MJ, Levin EJ, Hunt M, Yamada Y, Shippy AM, et al. (2008) Incidence of late rectal and urinary toxicities after three-dimensional conformal radiotherapy and intensity-modulated radiotherapy for localized prostate cancer. Int J Radiat Oncol Biol Phys 70: 1124-1129.

7. Wilkins A, Parker C (2010) Treating prostate cancer with radiotherapy. Nat Rev Clin Oncol 7: 583-589.

8. Levy Y, Paz A, Yosef RB, Corn BW, Vaisman B, et al. (2009) Biodegradable inflatable balloon for reducing radiation adverse effects in prostate cancer. $J$ Biomed Mater Res B Appl Biomater 91: 855-867.

9. Ben-Yosef R, Paz A, Levy Y, Alani S, Muncher Y, et al. (2009) A novel device for protecting rectum during prostate cancer irradiation: in vivo data on a large mammal model. J Urol 181: 1401-1406.

10. Livi L, Detti B, Meattini M, Sanchez LJ, Biti GP (2007) [Organ-confined prostate cancer: treatment with high doses of radioterapy (intensity modulated radiotherapy)]. Actas Urol Esp 31: 611-616.

11. Ryu JK, Winter K, Michalski JM, Purdy JA, Markoe AM, et al. (2002) Interim report of toxicity from $3 D$ conformal radiation therapy (3D-CRT) for prostate cancer on 3DOG/RTOG 9406, level III (79.2 Gy). Int J Radiat Oncol Biol Phys 54: 1036-1046.

12. Michalski JM, Winter K, Purdy JA, Parliament M, Wong H, et al. (2005) Toxicity after three-dimensional radiotherapy for prostate cancer on RTOG 9406 dose Level V. Int J Radiat Oncol Biol Phys 62: 706-713.

13. Kantzou I, Platoni K, Sandilos P, Gouliamos A, Kouvaris I, et al. (2011) Conventional versus virtual simulation for radiation treatment planning of prostate cancer: final results. J BUON 16: 309-315

14. Lawton CA, Michalski J, El-Naqa I, Buyyounouski MK, Lee WR, et al. (2009) RTOG GU Radiation oncology specialists reach consensus on pelvic lymph node volumes for high-risk prostate cancer. Int J Radiat Oncol Biol Phys 74 383-387.

15. Bentzen SM, Constine LS, Deasy JO, Eisbruch A, Jackson A, et al. (2010) Quantitative Analyses of Normal Tissue Effects in the Clinic (QUANTEC): an introduction to the scientific issues. Int J Radiat Oncol Biol Phys 76: S3-9.

16. Rohr K (1999) Extraction of 3d anatomical point landmarks based on invariance principles. Pattern Recognition 32: 3-15.

17. Markaki VE, Asvestas PA, Matsopoulos GK (2009) Application of Kohonen network for automatic point correspondence in $2 \mathrm{D}$ medical images. Comput Biol Med 39: 630-645.

18. Ingber L, Rosen B (1992) Genetic Algorithms and Very Fast Simulated Reannealing: A comparison. Mathematical and Computer Modelling 16: 87100.

19. Bookstein FL (1989) Principal warps: thin-plate splines and the decomposition of deformations. Pattern Analysis and Machine Intelligence, IEEE Transactions 11: $567-585$

20. Beard CJ, Kijewski P, BussiÃ re M, Gelman R, Gladstone D, et al. (1996) Analysis of prostate and seminal vesicle motion: implications for treatment planning. Int J Radiat Oncol Biol Phys 34: 451-458.

21. Antolak JA, Rosen II, Childress CH, Zagars GK, Pollack A (1998) Prostate target volume variations during a course of radiotherapy. Int J Radiat Oncol Biol Phys 42: 661-672.

22. Dawson LA, Mah K, Franssen E, Morton G (1998) Target position variability throughout prostate radiotherapy. Int J Radiat Oncol Biol Phys 42: 1155-1161.

23. D'Amico AV, Manola J, Loffredo M, Lopes L, Nissen K, et al. (2001) A practical method to achieve prostate gland immobilization and target verification for daily treatment. Int J Radiat Oncol Biol Phys 51: 1431-1436.

24. Wachter S, Gerstner N, Dorner D, Goldner G, Colotto A, et al. (2002) The influence of a rectal balloon tube as internal immobilization device on variations of volumes and dose-volume histograms during treatment course of conformal radiotherapy for prostate cancer. Int J Radiat Oncol Biol Phys 52: 91-100. 
Citation: Vassilis K, Matsopoulos G, Georgakopoulos J, Kalogeropoulos T, Platoni K, et al. (2013) Transperineal Implementation of Biocompatible Balloon in Patients Treated With Radiotherapy for Prostate Carcinoma: Feasibility and Quality Assurance Study in Terms of Anatomical Stabilization Using Image Registration Techniques. J Bioequiv Availab 5: 142-148. doi:10.4172/jbb.1000149

25. Teh BS, Mai WY, Uhl BM, Augspurger ME, Grant WH 3rd, et al. (2001) Intensity-modulated radiation therapy (IMRT) for prostate cancer with the use of a rectal balloon for prostate immobilization: acute toxicity and dose-volume analysis. Int J Radiat Oncol Biol Phys 49: 705-712.

26. Teh BS, Dong L, McGary JE, Mai WY, Grant W 3rd, et al. (2005) Rectal wall sparing by dosimetric effect of rectal balloon used during intensity-modulated radiation therapy (IMRT) for prostate cancer. Med Dosim 30: 25-30.

27. Ronson BB, Yonemoto LT, Rossi CJ, Slater JM, Slater JD (2006) Patient tolerance of rectal balloons in conformal radiation treatment of prostate cancer. Int J Radiat Oncol Biol Phys 64: 1367-1370.
28. Prada PJ, Fernandez J, Martinez AA, de la Rúa A, Gonzalez JM, et al. (2007) Transperineal injection of hyaluronic acid in anterior perirectal fat to decrease rectal toxicity from radiation delivered with intensity modulated brachytherapy or EBRT for prostate cancer patients. Int J Radiat Oncol Biol Phys 69: 95-102.

29. Prada PJ, Gonzalez H, Menendez C, Llaneza A, Fernandez J, et al. (2009) Transperineal injection of hyaluronic acid in the anterior perirectal fat to decrease rectal toxicity from radiation delivered with low-dose-rate brachytherapy for prostate cancer patients. Brachytherapy $8: 210-217$. 